\title{
TEMPERATURE AND TRANSVERSE EXPANSION OF FIREBALLS IN Pb+Pb COLLISIONS AT THE LHC
}

We analyze hadron transverse momentum spectra from $\mathrm{Pb}+\mathrm{Pb}$ Collisions recorded by the $\mathrm{LHC}$ detector ALICE. As the theoretical model we use a Monte Carlo model which includes production and decays of resonances. The transverse expansion of the fireball is shown to be stronger and the freeze-out temperature higher than in similar collisions at RHIC, where collision energy is more than ten times lower.

Keywords: Heavy ion collisions, quark-gluon plasma, transverse expansion, freeze-out temperature, particle spectra.

\section{Introduction}

Heavy atomic nuclei at room temperatures behave as liquids. If we collide two nuclei at moderate energies in a particle accelerator, the nuclei will be compressed and heated and as a result the nuclear liquid will turn into a hadron gas - a gas of pions, protons, neutrons and other hadron species. Hadrons include baryons (such as protons and neutrons) composed of three quarks, and mesons (such as pions and kaons) composed of one quark and one antiquark. The quarks are bound inside hadrons by the strong interaction which is mediated by gluons. Under normal conditions it is impossible to knock a single quark or gluon out of a hadron - the more we pull it, the stronger is the binding force which keeps it inside. This phenomenon is called confinement.

There is, however, a special state of nuclear matter, the so-called quark-gluon plasma (QGP), in which the quarks and gluons are not confined. Quantum chromodynamics (QCD) predicts that if the nuclei are heated above the critical temperature, the density of particles becomes so high that quarks are no longer bound to particular hadrons. Thus hadrons are dissolved into individual quarks and gluons. There is no confinement and quarks as well as gluons move freely within the whole QGP volume. Simulations of QCD on the lattice predict [1] that the (deconfinement) phase transition from the hadron gas phase to the QGP phase occurs at the critical temperature around $\mathrm{T}_{\mathrm{c}} \approx 170 \mathrm{MeV}$ and very high energy density. (In this paper we shall use energy units for the temperature. This should be understood as the energy equivalent which is obtained from the temperature by multiplying with the Boltzmann constant.) Such conditions are presently achieved in collisions of heavy nuclei at ultrarelativistic energies at two operating colliders: at the Relativistic Heavy Ion Collider (RHIC) of the Brookhaven National Laboratory gold nuclei are accelerated to the center of mass energies up to $200 \mathrm{GeV}$ per nucleon pair, and at the Large Hadron Collider (LHC) at CERN the QGP is formed in lead-lead collisions at the energy of $2760 \mathrm{GeV}$ per nucleon pair. The quark-gluon plasma is also believed to be the state of the Universe around $1 \mu$ s after the Big Bang.

Properties of the QGP and of the deconfinement phase transition are under intense theoretical and experimental scrutiny. The challenge of the program is the complicated nature of heavy ion collisions. The hot nuclear matter created in the collision (the fireball) expands and cools down very quickly and the QGP phase (if present) changes back into the hadron gas phase within $\sim 10^{-23} \mathrm{~s}$. The remaining hadronic fireball then continues its rapid expansion during which hadrons interact with each other and create new hadrons until the point of chemical freeze-out when the chemical composition of hadron species is fixed. After that the hadrons keep scattering elastically until the fireball disintegrates at the point of kinetic freeze-out after $\sim 10^{-22} \mathrm{~s}$. The initial information present in the QGP phase is thus heavily distorted by the phase transition into the hadron phase, expansion and hadron interactions. Fortunately some signals of QGP remain in the bulk properties of the collisions such as particle rates, transverse flow, chemical freeze-out temperature $T_{\text {ch }}$ and kinetic freeze-out temperature $T_{\text {kin }}$. These quantities can be studied via hadron spectra measured by the detectors. In this paper we will study transverse momentum $\left(p_{\mathrm{T}}\right)$ spectra of several hadron species measured by the ALICE detector at the LHC [2]

\footnotetext{
* ${ }^{12}$ Ivan Melo, ${ }^{2}$ Boris Tomasik

${ }^{1}$ Department of Physics, University of Zilina, Slovakia,

${ }^{2}$ Department of Physics, Matej Bel University Banska Bystrica, Slovakia

E-mail: melo@fyzika.uniza.sk
} 
and extract the transverse flow and $T_{\text {kin }}$ by fitting the experimental spectra with the predictions based on the Blast Wave model [3] which also includes the production of final state hadrons (e.g. pions) from the decays of short-lived resonances. The model is implemented as a Monte Carlo generator published under the title DRAGON [4].

\section{Transverse flow}

The fireball in the QGP phase exhibits collective flow/ expansion in the local thermal equilibrium which can be reasonably well described hydrodynamically. Expansion of the fireball results from strong pressure gradients in the hot nuclear matter. The fireball expands both longitudinally (along the direction of heavy ion beams, the $z$-axis) and transversely (perpendicularly to the beams).

The transverse flow is particularly interesting since it is entirely generated from the pressure of the hot matter unlike the longitudinal flow which is present also in the initial conditions (incident nucleons tend to continue in the beam direction after the collision). The transverse flow is described in terms of the transverse expansion velocity $v_{\mathrm{t}}$ which depends on the radial distance $r$ from the centre of the fireball. The outer layers of the fireball move with the largest velocity while the central part is at rest in the laboratory system.
In the Blast Wave model [3] one parametrizes the final freezeout state of the fireball. It is motivated by the assumption that the hydrodynamic relativistic expansion continues up to the point when the temperature of the fluid falls below $T_{\text {kin }}$ and the freezeout from a thermalized fluid into non-interacting free-streaming hadrons occurs suddenly. Within the model this happens at the same longitudinal proper time ${ }^{1} \tau=\sqrt{t^{2}-z^{2}}$ for each part of the fireball. Further assumptions include parametrization of $v_{\mathrm{t}}$ as

$$
v_{t}=\tanh \left(\sqrt{2} \eta_{f} \frac{r}{R}\right)
$$

where $\eta_{f}$ is the transverse flow parameter and $R$ is the radius of the cylindrical fireball at the freeze-out. Hadron number density is assumed to be constant within the cylinder and zero for $r>R$. The Blast Wave model gives a prescription to calculate transverse momentum $\left(p_{\mathrm{T}}\right)$ spectra of different species of hadrons as a function of $T_{\text {kin }}, \eta_{f}$, hadron masses and several other parameters. For more details about the model we refer the reader to the original works [3]. The calculated spectra can then be used to fit the experimental data and extract $T_{\text {kin }}$ and $\eta_{f}[2]$.

\section{DRAGON calculations and results}

We calculate the $p_{\mathrm{T}}$ spectra with the DRAGON tool based on the Blast Wave model to which a careful treatment of all 277

1 We work in units where the speed of light $\mathrm{c}=1$

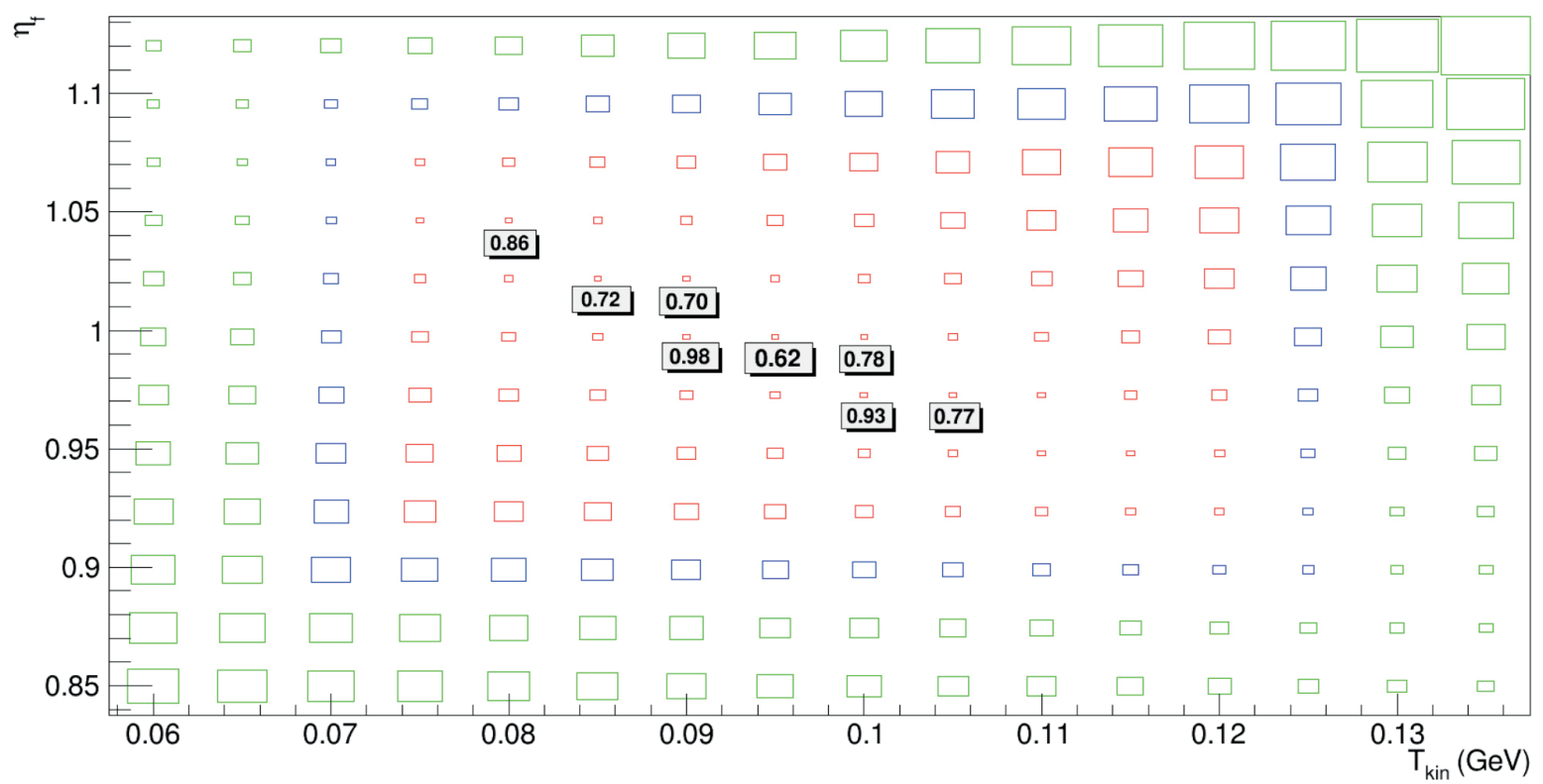

Fig. $1 \chi^{2}$ DRAGON fit to the $0-5 \%$ of the most central ALICE data. The size of the boxes is proportional to the $\chi^{2}$ value. The color of the boxes indicates the statistical size of the calculated spectra: green is based on 140 simulated events (collisions), blue on 1400 and red on 14000 events 
hadron species including decays of unstable resonances is added. We fix the chemical freeze-out temperature for the purpose of this study at $T_{\mathrm{ch}}=0.1656 \mathrm{GeV}$, baryochemical potential at $\mu_{\mathrm{B}}=0.002$ $\mathrm{GeV}$, strangeness potential ${ }^{2} \mu_{\mathrm{s}}=0.0069 \mathrm{GeV}$ as dictated by the chemical composition of the produced hadrons. Then, $T_{\text {kin }}$ and $\eta_{f}$ are varied in order to find the best fit to the data which include $p_{\mathrm{T}}$ spectra of six hadron species: protons, antiprotons, charged pions $\left(\pi^{+}, \pi\right)$, and charged kaons $\left(\mathrm{K}^{+}, \mathrm{K}^{-}\right)$. The best fit is given by the minimum of the $\chi^{2}$ distribution

$\chi^{2}\left(T_{k i n}, \eta_{f}\right)=\sum_{i=1}^{6} \sum_{j=1}^{j_{\max }} \frac{\left[N_{D R A G O N}^{\text {norm }}\left(i, j, T_{\text {kin }}, \eta_{f}\right)-N_{A L I C E}^{\text {norm }}(i, j)\right]^{2}}{\sigma_{A L I C E}^{\text {norm }}(i, j)^{2}}$

The first sum runs over the six species $i$, the second sum over all $p_{\mathrm{T}}$ bins $j$ (the maximum number of bins $j_{\max }=42,41,36$ for protons/antiprotons, pions and kaons respectively) with $\mathrm{p}_{\mathrm{T}}$ from 0.3 to $4.6 \mathrm{GeV}$ for protons/antiprotons, from 0.1 to 3.0 $\mathrm{GeV}$ for pions and from 0.2 to $3.0 \mathrm{GeV}$ for kaons [2]. $N_{\text {DRAGON }}$ $\left(N_{\text {ALICE }}\right)$ gives the normalized numbers of hadrons of $i$-th species in the $j$-th bin for DRAGON and ALICE respectively, $\sigma$ is the experimental error given by the combination of the statistical and systematic errors. Each of the six hadron spectra is normalized independently in order to match the total numbers of each species between DRAGON and ALICE.

2 Nonzero strangeness potential leads to unequal numbers of strange hadrons and antihadrons, such as $\mathrm{K}$ - and $\mathrm{K}+$ (strange hadrons contain at least one strange quark which is one of six quarks in the Standard model of particle physics),
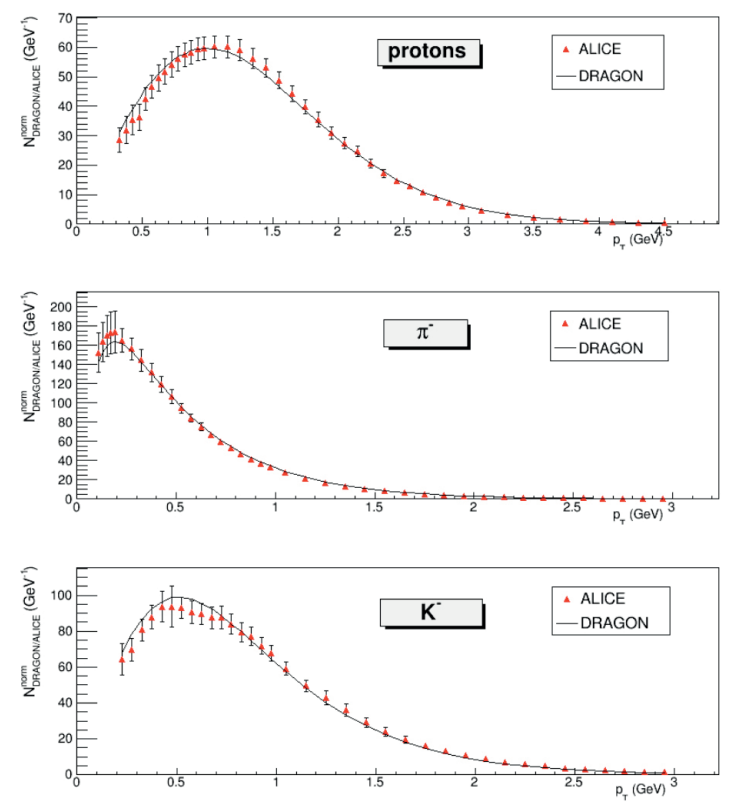

Fig. 2 The transverse momentum $\left(p_{T}\right)$ spectra from central collisions for six hadron species calculated with DRAGON at the $\chi^{2}$ minimum (solid line) and compared to 0-5\% most central Pb+Pb experimental data from ALICE (red triangles)
The resulting $\chi^{2}$ divided by the number of degrees of freedom $\left(\mathrm{N}_{\mathrm{dof}}=232\right)$ from $0-5 \%$ most central $\mathrm{Pb}+\mathrm{Pb}$ collisions (a central collision is one with the zero impact parameter between the two nuclei) are shown in Fig. 1 as a function of $T_{\text {kin }}$ and $\eta_{f}$. The area of the boxes is proportional to the $\chi^{2}$ value, the color of the boxes indicates the statistical size of the calculated spectra: green is based on 140 simulated events (collisions), blue on 1400 and red on 14000 events. The minimum $\chi^{2} / \mathrm{N}_{\text {dof }}$ value, 0.62 , lies at $T_{\text {kin }}=$ $0.095 \mathrm{GeV}$ and $\eta_{f}=1.0$.

The transverse momentum $\left(p_{\mathrm{T}}\right)$ spectra for the six hadron species simulated with DRAGON at the minimum and compared to $0-5 \%$ most central $\mathrm{Pb}+\mathrm{Pb}$ experimental data are shown in Fig. 2. Fit results also for other centralities are summarized in Table 1.

Freeze-out temperatures and transverse expansion parameters from the fits to transverse momentum $\left(\mathrm{p}_{\mathrm{T}}\right)$ spectra at different centralities based on 1400 simulated events

Table 1

\begin{tabular}{|c|c|c|c|}
\hline Centrality & $T_{\text {kin }}[\mathrm{MeV}]$ & $\eta_{\mathrm{f}}$ & $\chi^{2} / N_{\text {dof }}$ \\
\hline $0-5 \%$ & 95 & 1 & 0.673 \\
\hline $5-10 \%$ & 95 & 1 & 0.764 \\
\hline $10-20 \%$ & 105 & 0.975 & 0.733 \\
\hline $20-30 \%$ & 120 & 0.925 & 0.881 \\
\hline $30-40 \%$ & 125 & 0.9 & 1.044 \\
\hline $40-50 \%$ & 145 & 0.825 & 1.411 \\
\hline $50-60 \%$ & 155 & 0.775 & 1.900 \\
\hline
\end{tabular}
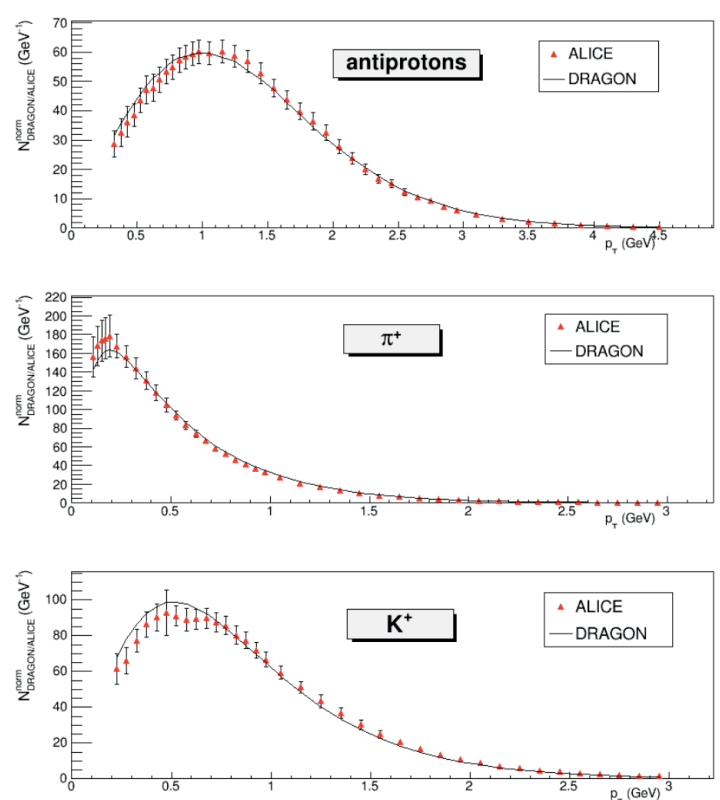


\section{COMMNICOIIIONS}

To make a comparison with lower energy data from RHIC we repeated the fit with the $0-5 \%$ of the most central STAR data at the energy $62.4 \mathrm{GeV}$ per nucleon pair [5] and found the minimum $\chi^{2}$ value at $T_{\text {kin }}=0.085 \mathrm{GeV}$ and $\eta_{f}=0.825$. We observe that going from RHIC to the LHC energy both the freeze-out temperature and the transverse flow go up. This can be understood as a result of higher pressure which builds up at the LHC heavy ion collisions. Higher pressure leads to stronger transverse flow and stronger expansion also means that the interaction rate of hadrons in the fireball drops below the expansion rate earlier, at higher freeze-out temperature. ALICE has published similar results [2], however, our study properly accounts for all hadron species including resonance decays.

\section{Acknowledgments}

We would like to thank Mateusz Ploskon, ALICE Deputy Physics Coordinator, for supplying us with the ALICE $p_{\mathrm{T}}$ spectra. We would also like to acknowledge the support by APVV-0050-11 and VEGA 1/0457/12.

\section{References}

[1] AOKI, Y. et al.: Phys. Lett. B 643, 2006, 46.

[2] "Centrality Dependence of $\mathrm{p}, \mathrm{K}, \mathrm{p}$ Production in Pb-Pb Collisions at $\sqrt{\mathrm{s}_{\mathrm{NN}}}=2.76 \mathrm{TeV}$ ", ALICE Collaboration, [arXiv:1303.0737 [hep-ex]].

[3] SCHNEDERMANN, E., SOlLFRANK, J., HEINZ, U.: Phys. Rev. C 48, 1993, 2462. [arXiv:nucl-th/9307020]; P. J. Siemens, J. O. Rasmussen, Phys. Rev. Lett., 42, 1979, 880.

[4] TOMASIK, B.: Comp. Phys. Commun. 180, 2009, 1642- 1653.

[5] ABELEV, B. I. et al, STAR Collaboration, Phys. Rev. C 79 (2009) 034909. 